\title{
Sequential bilateral retinal artery occlusion
}

This article was published in the following Dove Press journal:

Clinical Ophthalmology

II April 2014

Number of times this article has been viewed

\author{
Noel Padrón-Pérez' \\ Janny Rosario Aronés ${ }^{2}$ \\ Silvia Muñoz' \\ Luis Arias-Barquet' \\ Jorge Arruga ${ }^{1,3}$ \\ 'Department of Ophthalmology, \\ Hospital Universitari de Bellvitge, \\ ${ }^{2}$ Hospital de l'Esperança - Parc de \\ Salut Mar, ${ }^{3}$ Institut Català de Retina, \\ Barcelona, Spain
}

Correspondence: Noel Padrón-Pérez Department of Ophthalmology, Hospital Universitari de Bellvitge, Feixa Llarga L'Hospitalet de Llobregat, Barcelona 08097, Spain

Tel +34932607600

Fax +34932607533

Email noelpdrn@gmail.com

\begin{abstract}
An 86 year old woman experienced a sequential bilateral loss of vision over a period of less than 24 hours. Clinical findings and complementary studies suggested a bilateral atherogenic embolic event. Initially, she presented a superior branch retinal artery occlusion in her right eye followed by a central retinal artery occlusion with cilioretinal artery sparing in her left eye. Some conservative maneuvers performed did not improve visual acuity in the left eye. Supra-aortic Doppler ultrasonography revealed mild right internal carotid artery stenosis and moderate left internal carotid artery stenosis with a small, smooth, and homogeneous plaque. The transthoracic echocardiography showed a severe calcification of the mitral valve with a mild-moderate rim of stenosis. Central retinal artery occlusion and branch retinal artery occlusion are characterized by painless monocular loss of vision. Clinical approach and management attempt to treat the acute event, find the source of the vascular occlusion, and prevent further vascular events from occurring. Giant cell arteritis is a potentially treatable cause of central retinal artery occlusion and should be excluded in every single patient over 50 years old.
\end{abstract}

Keywords: loss of vision, branch retinal artery occlusion, central retinal artery occlusion, Hollenhorst plaque

\section{Introduction}

Central retinal arterial occlusion (CRAO) and branch retinal artery occlusion (BRAO) are considered forms of stroke, with similar clinical approach and management. CRAO is a serious ophthalmologic disease with a poor prognosis. The incidence of acute CRAO in the US is 8.5 per 100,000 people..$^{1,2}$ Demographic characteristics of patients with CRAO and BRAO are consistent with those seen for other vascular disorders. The mean age of patients is between 60 and 65 years. ${ }^{3,4}$ More than $90 \%$ of patients with CRAO and BRAO are over the age of 40 years, although individuals as young as 12 years have been affected. ${ }^{3,5,6}$ Men are more commonly affected than women. Hypertension, smoking, and diabetes are more prevalent among patients with retinal artery occlusion (RAO) compared with controls. ${ }^{7,8}$ Patients presenting with CRAO often have previously undiagnosed vascular risk factors.

We report a case of sequential bilateral artery occlusion over a period of less than 24 hours, presenting initially with permanent BRAO followed by nonarteritic CRAO with cilioretinal artery sparing.

\section{Case presentation}

An 86-year-old woman presented at the emergency room with sudden painless loss of visual field in her right eye (oculus dexter [OD]). Past medical history included primary 
systemic hypertension and hypercholesterolemia. She had undergone cataract surgery in both eyes (oculi uterque [OU]) 2 years previously. The patient had also experienced previous episodes of transient vision loss in her left eye (oculus sinister [OS]). Best-corrected visual acuity (BCVA) was 20/25 in OU. A confrontation test proved lower visual field defect in the OD. Intraocular pressure was normal, and anterior segment examination just revealed bilateral mild posterior capsular opacification. Fundoscopy disclosed superior BRAO in the OD, with a Hollenhorst plaque at the first bifurcation of the superior temporal arterial branch, attenuation, and "boxcarring" of the blood column in the involved region (Figure 1A). Small and medium-sized hard drusen were observed in OU. Oral antiplatelet therapy and rigorous follow-up of hypertension and hypercholesterolemia were recommended. Fourteen hours after discharge, the patient came back to the emergency room with sudden complete visual loss in the OS that had started 4 hours earlier. BCVA was 20/25 in the OD and hand motion in the OS, and there was a relative afferent pupillary defect in the OS. Fundus examination disclosed CRAO with cilioretinal artery sparing in the OS (Figure 1B). A cherryred spot was observed in the macula. Fundoscopy of the OD showed previous BRAO with a small embolus after the second bifurcation of the superior temporal retinal vessel (Figure 1a'). Spectral domain optical coherence tomography of the macula (Figure 2) showed a hyperreflective image in the OS, suggesting swelling of internal layers of the retina, and revealed $366 \pm 38 \mu \mathrm{m}$ of foveal thickness (more prominent in the nasal quadrants). Fluorescein angiography showed impaired filling of the superior temporal arterial branch and branches of the central retinal artery (CRA) in the right and left eyes, respectively (Figure 3).

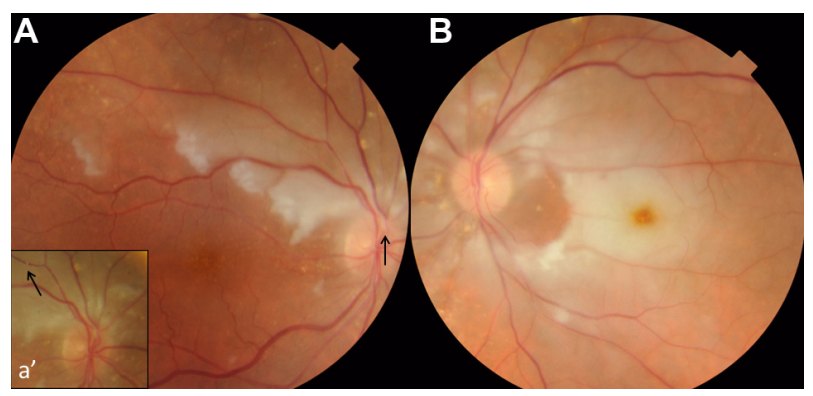

Figure I (A and B) Fundus photographs show superior temporal branch retinal artery occlusion in the OD and central retinal artery occlusion with cilioretinal artery sparing in the OS. (A) Morphological appearance suggests a Hollenhorst plaque at the first bifurcation of the temporal superior arterial vessel (black arrow). (B) A cherry-red spot can be seen against the pale retina. Attenuation and "boxcarring" of the blood column in the involved region were observed in OU. After the second bifurcation of the superior temporal retinal vessel, a small embolus (or fragment of the first one) is observed (a').

Abbreviations: OD, oculus dexter (right eye); OS, oculus sinister (left eye); $\mathrm{OU}$, oculi uterque (both eyes).

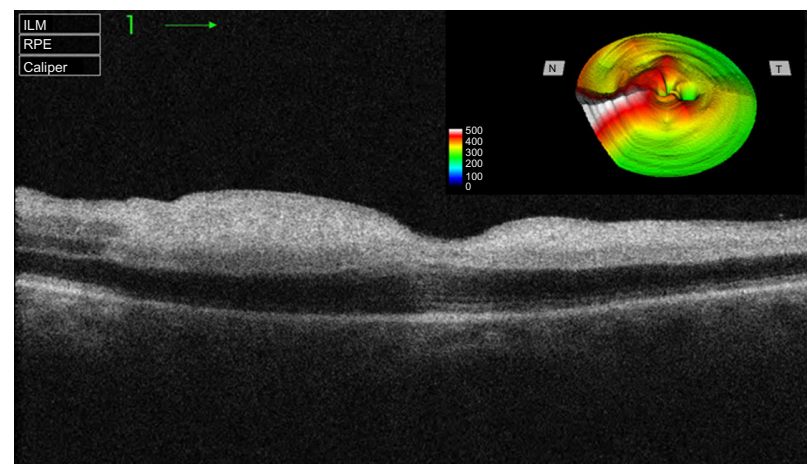

Figure 2 Spectral domain optical coherence tomography of the macula in the OS reveals a hyperreflective image corresponding to intracellular edema of the internal layers of the retina $(366 \pm 38 \mu \mathrm{m}$ of foveal thickness), more prominent in the nasal quadrants.

Abbreviations: OS, oculus sinister (left eye); ILM, internal limiting membrane; RPE, retinal pigment epithelium.

Despite some conservative maneuvers performed (paracentesis, ocular massage, intraocular hypotensive agents), there was no improvement of VA in the OS. Electrocardiogram results, erythrocyte-sedimentation rate, and serum $\mathrm{C}$-reactive protein were completely normal in the acute phase. There was no evidence of systemic symptoms related to Horton's disease. Systemic workup was completed by internal medicine and a cardiologist.

Supra-aortic Doppler ultrasonography revealed mild right internal carotid artery stenosis (between $0 \%$ and $15 \%$ ) ipsilateral to the eye with BRAO. There was moderate left internal carotid artery stenosis (between 15\% and 50\%), with a small, smooth, homogeneous plaque in the side of the CRAO. Transthoracic echocardiography revealed a severe calcification of the mitral valve with a mild-moderate rim of stenosis. The patient was informed about these findings and the high risk for cardiovascular disease, myocardial infarction and stroke.

Considering exploration and complementary studies, our patient experienced a sequential bilateral atherogenic

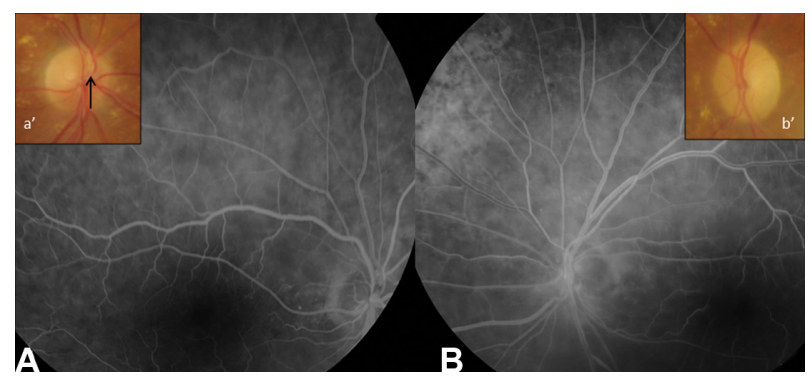

Figure 3 Fluorescein angiography (A and $\mathbf{B}$ ) shows slowed filling of the superior temporal arterial branch in the OD and branches of the central retinal artery in the OS. The optic disk became pale in OU ( $a^{\prime}$ and $\left.b^{\prime}\right) 3$ months after bilateral retinal artery occlusion. The OD presented a pale aspect of the optic disk in the upper and temporal quadrants $\left(a^{\prime}\right)$, and the OS showed diffuse atrophy $\left(b^{\prime}\right)$.

Abbreviations: OD, oculus dexter (right eye); OS, oculus sinister (left eye); OU, oculi uterque (both eyes). 
embolic event (BRAO in the OD and nonarteritic CRAO with cilioretinal artery sparing in the OS). The patient was evaluated every month to monitor progression. Three months after BRAO, visual function remained stable, and both optic disks appeared pale (Figure 3a' and $b^{\prime}$ ).

\section{Discussion}

CRAO and BRAO present with acute, painless loss of monocular vision. BRAO may be permanent or transient. It is usually due to embolism, hemodynamic causes, spasm induced by serotonin, and occasionally vasculitis. ${ }^{9}$ In BRAO, there is segmental retinal infarction in the region supplied by the occluded branch retinal arteriole; there is frequently attenuation and boxcarring of the blood column in the vessels in the involved region, with or without the presence of an embolus. Our case presented with a visual field defect in the OD, and fundoscopy revealed a permanent BRAO with a visible embolus at the superior arterial vessel.

The location of BRAO obviously determines the location and type of visual field defect. BCVA in the present case remained at 20/25, with a permanent visual field defect in the OD. In a study based on 199 consecutive untreated patients (212 eyes) $)^{10}$ in eyes with permanent BRAO (133), initial VA was $20 / 40$ or better in $74 \%$, with central scotoma in $20 \%$, central inferior altitudinal defect in $13 \%$, and inferior nasal and superior sector defects in $29 \%$ and $24 \%$, respectively. Of those with follow-up, in the eyes with VA worse than 20/40, it improved in $79 \%$ (eleven of 14 ), abnormal central visual field defect improved in $47 \%$, and abnormal peripheral visual field defect improved in 52\%.

Retinal emboli are made of cholesterol in $74 \%$, calcific material in $10.5 \%$, and platelet/fibrin in only $15.5 \%$ of cases. ${ }^{11}$ Cholesterol emboli typically appear as a more yellowish, refractile plaque. Digital pressure on the eye can make them turn within the vessel, causing them to become more or less visible to the examiner.

Platelet-fibrin emboli appear as whitish-gray, nonreflective plugs that are mobile. They may appear in "showers", and may pass through without causing an occlusion. They are usually associated with mural thrombus in the carotid artery or cardiac valvular structures. ${ }^{11}$ There was no evidence of mural thrombus in the carotid artery or cardiac valvular structures in the present case.

Calcific emboli appear as large, yellowish-white, nonreflective plaques. They more likely are found in the larger arterioles near the optic disk. They are associated with calcified cardiac valves and atheromatous plaques of the carotid artery. ${ }^{11}$
It is necessary to point out that the presence of ipsilateral emboli suggests ipsilateral vascular disease, and the presence of bilateral emboli a cardiac or aortic source. ${ }^{12}$ Vascular and cardiac diseases were present in our case as sources of emboli. The emboli in the OD seemed to correspond to a Hollenhorst plaque, which may suggest the carotid artery as the source. Systemic evaluation and morphological appearance of emboli seen in BRAO may indistinctly suggest the three types of emboli.

One study demonstrated that attempts to categorize emboli into cholesterol, calcific, or other by fundoscopic examination had large intraobserver and interobserver variability. ${ }^{13}$ The authors recommended that systemic evaluation not be based on qualitative assessment of the type of emboli.

Patients with cholesterol emboli often have a concurrent history of hypertension, diabetes, carotid artery disease, peripheral vascular disease, hypercholesterolemia, hyperlipidemia, and/or atherosclerosis. The patient may have previously experienced transient episodes of monocular blindness (as in the present case). ${ }^{7,8,14-16}$

The management of BRAO depends upon the type. Although some procedures have been advocated to treat permanent BRAO, there is no strong evidence to support them. The apparent efficacy of surgical embolectomy ${ }^{17}$ simply represents the natural history of disease. Concerning translumenal neodymium-doped yttrium aluminum garnet (Nd:YAG) embolysis/embolectomy, ${ }^{18}$ the possible benefit of this procedure could be achieved within 2 or 3 hours of the onset of visual loss, while the retina is still not irreversibly damaged by acute ischemia.

Our case presented nonarteritic CRAO with cilioretinal artery sparing; BCVA in the affected eye was hand motion. In a prospective study of 244 patients ( 360 eyes) with CRAO, nonarteritic CRAO with cilioretinal artery sparing was seen in $14 \%$ of cases. ${ }^{19}$ In 35 eyes, the initial VA overall was $20 / 30$ or better in $29 \%, 20 / 60-20 / 100$ in $14 \%, 20 / 200$ in $6 \%$, counting fingers in $20 \%$, hand motion in $26 \%$, and light perception in $6 \%$ of cases. In CRAO, if the patent cilioretinal artery supplies the foveal region, visual acuity (VA) is almost always normal, with an intact central island visual field corresponding to the distribution of the artery. However, if it supplies only a small peripapillary region (as in the present case) or retina nasal to the disk, a small island visual field may be present, with poor VA. ${ }^{9}$

The classical ophthalmoscopic findings of acute and late CRAO are well known, but there is limited information concerning the prevalence and temporal development of the fundus findings. A prospective study based on 248 eyes 
with CRAO described the pattern and evolution of fundus findings in the four types of CRAO. ${ }^{20}$ The main findings during the initial examination for permanent CRAO were retinal opacity in the posterior pole $(58 \%)$, cherry-red spot (90\%), boxcarring (19\%), retinal arterial attenuation (32\%), and optic disk edema (22\%), and pallor (39\%). The fovea assumes a cherry-red spot because of a combination of two factors: 1) the intact retinal pigment epithelium and choroid underlying the fovea, and 2) the foveolar retina is nourished by the choriocapillaris.

Retinal tolerance to acute ischemia is essential to understanding the RAO disorders and their management. An important study performed in 38 elderly, atherosclerotic, and hypertensive rhesus monkeys (mimicking patients with CRAO) suggested that CRAO lasting for about 240 minutes results in massive and irreversible retinal damage. There was no apparent morphometric evidence of damage with CRAO of less than 97 minutes, but occlusion of 105 minutes but less than 240 minutes produced a variable degree of damage, while occlusion for 240 minutes or more produced total optic nerve atrophy and nerve-fiber damage. ${ }^{21}$ According to these facts, treatment instituted at any time beyond 4 hours after the onset of CRAO cannot have any scientific rationale for improvement of vision.

Conventional advocated treatments for CRAO include ocular massage in an effort to dislodge the embolus in the CRA; a reduction of intraocular pressure by various medical and surgical means; vasodilation of the CRA by sublingual isosorbide dinitrate, rebreathing of expired $\mathrm{CO}_{2}$ in a bag, or breathing carbogen or retrobulbar vasodilators; and antiplatelet and heparin therapy. Other miscellaneous treatments include isovolemic hemodilution; hyperbaric oxygen; reduction of red blood cell rigidity by giving pentoxifylline, systemic steroids intravenously to reduce vascular endothelial edema following CRAO, Nd:YAG laser arteriotomy and embolectomy; and cannulation of the supraorbital artery and retrograde injection of antispasmodic papaverine. ${ }^{9}$

The intra-arterial fibrinolysis delivered directly into the ophthalmic artery by superselective administration of a thrombolytic agent has gained popularity in the past few years. An extensive and critical review of the published studies shows several fundamental problems, including: 1) almost all studies have been retrospective, 2) there have been almost no randomized, controlled, masked studies, 3 ) there is no fluorescein fundus angiographic evidence to document improved blood flow immediately after therapy, 4) a lack of comparison with a satisfactory natural history control, and 5) CRAO is not classified into various types to determine the visual outcome.
In a prospective randomized multicenter clinical trial to compare treatment outcome after conservative standard management and local intra-arterial fibrinolysis (LIF) using recombinant tissue plasminogen activator for acute nonarteritic CRAO, there was similar efficacy concerning BCVA in both groups, but a high rate of adverse reactions in the LIF group was observed. The study was stopped after the first interim analysis. The researchers concluded that they could not recommend LIF for the management of acute CRAO. The mean interval between first symptoms and therapy was $10.99 \pm 5.49$ hours. $^{22}$

Anh et $\mathrm{al}^{23}$ revealed in a recent study that in subtotal and total CRAO, there were no significant differences in visual outcomes between groups, but LIF may provide early restoration of retinal perfusion and offer functional benefits in the management of incomplete CRAO. LIF resulted in clinically insignificant cerebral infarcts, detectable on brain imaging in $8 \%$ of patients, without hemorrhagic transformation.

Some conventional procedures were performed in the present case in an effort to dislodge the embolus in the CRA, even in the knowledge that almost all the advocated modes of treatments claiming VA improvement have been performed far longer than 4 hours after onset, which indicates that those results simply reflected natural history.

Murphy-Lavoie et $\mathrm{al}^{24}$ have proposed that in patients with a clinical picture of CRAO who present within 24 hours of vision loss, supplemental oxygen should be started immediately at the highest possible fraction of inspired oxygen. If vision is not quickly restored, emergent hyperbaric oxygen should be undertaken if feasible. If the patient responds to hyperbaric oxygen, follow-up treatment with supplemental oxygen should be customized to maintain retinal viability until the obstructed retinal artery reanalyzes. Two comments regarding this modality of treatment: 1) the viability of a damaged retina cannot be restored after 240 minutes of ischemia, even with high fractions of inspired oxygen or hyperbaric oxygen, and 2) there are various types of acute RAO disorders. CRAO has been described as all one clinical entity; the same applies to BRAO. Based on their pathogeneses and clinical features, CRAO and BRAO each consist of multiple distinct clinical entities. Understanding these classifications and pathogeneses is essential for full comprehension of the various aspects of these disorders and their management.

A "shower of emboli" could be considered in our case which presented as bilateral and sequential embolic event occurring within a 24 hour period. Cholesterol-embolization syndrome, aortic aneurysms, abdominal aortic aneurysm repair, severe aortic disease, carotid endarterectomy, patients undergoing angiography or who receive percutaneous 
coronary transluminal angioplasty vein grafts, or patients undergoing renal artery angioplasty or cardiac catheterization should be included as differential diagnoses. After a rigorous systemic evaluation, all of the aforementioned conditions were ruled out.

A brief comment concerning cholesterol-embolization syndrome may be appropriate. The diagnosis of this condition must be considered in patients older than 50 years who have atherosclerotic disease presenting with multisystem dysfunction after undergoing an invasive vascular procedure or receiving an anticoagulant or thrombolytic agent within the past several months. All patients with the classic triad of livedo reticularis, acute renal failure, and eosinophilia should be evaluated for cholesterol-embolization syndrome, including a fundoscopic examination. ${ }^{25}$

The carotid artery and the heart are the sources of embolism to the retinal arteries. The majority of retinal occlusions are due to relatively small emboli. These emboli may be derived from nonhemodynamically significant obstruction, because it requires only a small plaque(s), which may be present with or without any significant carotid artery stenosis. The presence of plaques in the carotid artery is generally of much greater importance than the degree of stenosis in the artery. ${ }^{9,15}$

Carotid Doppler ultrasonography showed stenosis between $1 \%$ and $15 \%$ (without visible plaques) in the right internal carotid artery, ipsilateral to the eye with BRAO, and moderate left internal carotid artery stenosis (between $15 \%$ and $50 \%$ ), with a small, smooth, homogeneous, visible plaque ipsilateral to the eye with CRAO. When evaluating the results of carotid Doppler study for ocular microembolism, the absence of marked carotid artery stenosis on Doppler does not rule out ocular ischemia. Vascular insufficiency in the eye caused by a significant carotid artery stenosis is seen only rarely, and occurs primarily in ocular ischemic syndrome, but not all patients with ocular ischemia show severe carotid artery stenosis or occlusion. ${ }^{9,15}$ Also, we must keep in mind that carotid Doppler imaging evaluates the artery in the neck only and not above and below that, so the source of microembolism may be at those locations.

Transthoracic echocardiography evidenced a severe calcification of the mitral valve. These findings may represent another source of emboli. Normal echocardiography does not rule out the heart as the source of retinal microembolism; its resolution or the technique used may not be sensitive enough to detect very small valvular or other cardiac lesions.

In a cohort study reported by Hayreh, ${ }^{9}$ abnormal echocardiography of an embolic source was present in 52\% of patients with nonarteritic CRAO. Of those with an embolic source, $26 \%$ were from the mitral valve, $38 \%$ from the aortic valve, and $36 \%$ from both mitral and aortic valves. The mitral valve lesions comprised 57\% calcified valve, $17 \%$ mitral valve prolapse, and $26 \%$ other types of lesions. The aortic valve lesions were $78 \%$ calcified valve and $22 \%$ other types. This finding of an abnormal echocardiogram with an embolic source was not significantly associated with cerebrovascular accident/transient ischemic attack or ischemic heart disease. The same study reported that in relating carotid Doppler findings to echocardiography findings, an abnormal echocardiogram with embolic source is present in $62 \%$ of those with plaque in CRAO and in 44\% with BRAO. In such cases, the embolus could have come from the carotid artery, the heart, or possibly both.

\section{Conclusion}

Sequential nonarteritic RAO occurring within a 24 hour period is an extremely rare condition. CRAO has a poor prognosis for spontaneous recovery of vision. Efforts to restore vision should begin emergently. Unfortunately, none of the proposed treatments has so far stood the test of time, in spite of enthusiastic claims. A beneficial effect is possible in acute RAO disorder if some of the aforementioned treatments are started aggressively much less than 4 hours from the onset. Prospective and randomized studies with an appropriate methodology are necessary in order to get conclusions concerning the efficacy of the different therapeutic options.

There are various types of acute RAO disorders. Understanding these classifications and pathogeneses is essential for full comprehension of the various aspects of these entities and their management. Since we now understand their pathogeneses, we should be better able to explore the management of the different clinical disorders individually rather than lumping diverse conditions into one group.

In persons aged 50 years and older, it is essential to rule out giant cell arteritis immediately, and if there is any suspicion of that, the patient should be immediately started on high-dose steroid therapy to prevent catastrophic visual loss. Diagnostic workup in CRAO and BRAO focuses on identifying the underlying etiology, in order to institute appropriate secondary prevention measures to reduce the risk of future vascular events.

\section{Disclosure}

The authors report no conflicts of interest in this work.

\section{References}

1. Rumelt S, Dorenboim Y, Rehany U. Aggressive systematic treatment for central retinal artery occlusion. Am J Ophthalmol. 1999;128(6): $733-738$ 
2. Leavitt JA, Larson TA, Hodge DO, Gullerud RE. The incidence of central retinal artery occlusion in Olmsted County, Minnesota. Am J Ophthalmol. 2011;152(5):820-823.

3. Yuzurihara D, Iijima H. Visual outcome in central retinal and branch retinal artery occlusion. Jpn J Ophthalmol. 2004;48(5):490-492.

4. Smit RL, Baarsma GS, Koudstaal PJ. The source of embolism in amaurosis fugax and retinal artery occlusion. Int Ophthalmol. 1994; $18(2): 83-86$.

5. Karjalainen K. Occlusion of the central retinal artery and retinal branch arterioles. A clinical, tonographic and fluorescein angiographic study of 175 patients. Acta Ophthalmol Suppl. 1971;109:1-95.

6. Warrasak S, Tapaneya-Olarn W, Euswas A, Sriphojanart S, Sirikulchayanonta V, Leelachaikul P. Fibromuscular dysplasia: a rare cause of cilioretinal artery occlusion in childhood. Ophthalmology. 2000;107(4):737-741.

7. Wong TY, Larsen EK, Klein R, et al. Cardiovascular risk factors for retinal vein occlusion and arteriolar emboli: the Atherosclerosis Risk in Communities and Cardiovascular Health studies. Ophthalmology. 2005;112(4):540-547.

8. Wong TY, Klein R. Retinal arteriolar emboli: epidemiology and risk of stroke. Curr Opin Ophthalmol. 2002;13(3):142-146.

9. Hayreh SS. Acute retinal arterial occlusive disorders. Prog Retin Eye Res. 2011;30(5):359-394.

10. Hayreh SS, Podhajsky PA, Zimmerman MB. Branch retinal artery occlusion: natural history of visual outcome. Ophthalmology. 2009; 116(6):1188-1194.

11. Arruga J, Sanders MD. Ophthalmologic findings in 70 patients with evidence of retinal embolism. Ophthalmology. 1982;89(12):1336-1347.

12. Petzold A, Islam N, Hu HH, Plant GT. Embolic and nonembolic transient monocular visual field loss: a clinicopathologic review. Surv Ophthalmol. 2013;58(1):42-62.

13. Sharma S, Pater JL, Lam M, Cruess AF. Can different types of retinal emboli be reliably differentiated from one another? An inter- and intraobserver agreement study. Can J Ophthalmol. 1998;33(3):144-148.
14. Wong TY, Klein R. Retinal arteriolar emboli: epidemiology and risk of stroke. Curr Opin Ophthalmol. 2002;13(3):142-146.

15. Hayreh SS, Podhajsky PA, Zimmerman MB. Retinal artery occlusion: associated systemic and ophthalmic abnormalities. Ophthalmology. 2009;116(10):1928-1936.

16. Babikian V, Wijman CA, Koleini B, Malik SN, Goyal N, Matjucha IC. Retinal ischemia and embolism. Etiologies and outcomes based on prospective study. Cerebrovasc Dis. 2001;12(2):108-113.

17. García-Arumí J, Martinez-Castillo V, Boixadera A, Fonollosa A, Corcostegui B. Surgical embolus removal in retinal artery occlusion. Br J Ophthalmol. 2006;90(10):1252-1255.

18. Opremcak E, Rehmar AJ, Ridenour CD, Borkowski LM, Kelley JK. Restoration of retinal blood flow via translumenal Nd:YAG embolysis/ embolectomy (TYL/E) for central and branch retinal artery occlusion. Retina. 2008;28(2):226-235.

19. Hayreh SS, Zimmerman MB. Central retinal artery occlusion: visual outcome. Am J Ophthalmol. 2005;140(3):376-391.

20. Hayreh SS, Zimmerman MB. Fundus changes in central retinal artery occlusion. Retina. 2007;27(3):276-289.

21. Hayreh SS, Zimmerman MB, Kimura A, Sanon A. Central retinal artery occlusion. Retinal survival time. Exp Eye Res. 2004;78(3):723-736.

22. Schumacher M, Schmidt D, Jurklies B, et al. Central retinal artery occlusion: local intra-arterial fibrinolysis versus conservative treatment, a multicenter randomized trial. Ophthalmology. 2010;117(7):1367-1375.

23. Ahn SJ, Kim JM, Hong JH, et al. Efficacy and safety of intra-arterial thrombolysis in central retinal artery occlusion. Invest Ophthalmol Vis Sci. 2013;54(12):7746-7755.

24. Murphy-Lavoie H, Butler F, Hagan C. Central retinal artery occlusion treated with oxygen: a literature review and treatment algorithm. Undersea Hyperb Med. 2012;39(5):943-953.

25. Kronzon I, Saric M. Cholesterol embolization syndrome. Circulation. 2010;122(6):631-641.
Clinical Ophthalmology

\section{Publish your work in this journal}

Clinical Ophthalmology is an international, peer-reviewed journal covering all subspecialties within ophthalmology. Key topics include: Optometry; Visual science; Pharmacology and drug therapy in eye diseases; Basic Sciences; Primary and Secondary eye care; Patient Safety and Quality of Care Improvements. This journal is indexed on Submit your manuscript here: http://www.dovepress.com/clinical-ophthalmology-journal

\section{Dovepress}

PubMed Central and CAS, and is the official journal of The Society of Clinical Ophthalmology (SCO). The manuscript management system is completely online and includes a very quick and fair peer-review system, which is all easy to use. Visit http://www.dovepress.com/ testimonials.php to read real quotes from published authors. 